\title{
Renal Pharmacology
}

National Cancer Institute

\section{Source}

National Cancer Institute. Renal Pharmacology. NCl Thesaurus. Code C18996.

The science concerned with drugs and their actions and uses in kidney biology and the treatment of kidney disease. 